\title{
Dislocation core structures in Si-doped GaN
}

S. L. Rhode, \#, M. K. Horton ${ }^{2}$, W. Y. Fu ${ }^{1}$, S. -L. Sahonta ${ }^{1}$, M. J. Kappers ${ }^{1}$, T. J. Pennycook ${ }^{3,}$ ${ }^{4}$, C. J. Humphreys ${ }^{1}$, R. O. Dusane ${ }^{5}$, M. A. Moram ${ }^{1,2}$

1) Dept. Materials Science \& Metallurgy, University of Cambridge, Charles Babbage Road, Cambridge, CB3 OFS, UK.

2) Dept. Materials, Imperial College London, Exhibition Road, London, SW7 2AZ, UK.

3) SuperSTEM, STFC Daresbury Laboratories, Warrington, WA4 4AD, UK.

4) Department of Materials, University of Oxford, Parks Road, Oxford, OX1 3PH, UK.

5) Dept. Metallurgical Engineering and Materials Science, Indian Institute of Technology Bombay, Mumbai, 400076, India.

\#srhode@imperial.ac.uk

\begin{abstract}
:
Aberration-corrected scanning transmission electron microscopy was used to investigate the core structures of threading dislocations in plan-view geometry of $\mathrm{GaN}$ films with a range of Si-doping levels and dislocation densities ranging between $(5 \pm 1) \times 10^{8}$ and $(10 \pm 1) \times 10^{9}$ $\mathrm{cm}^{-2}$. All $a$-type (edge) dislocation core structures in all samples formed 5/7-atom ring core structures, whereas all $(a+c)$-type (mixed) dislocations formed either double 5/6-atom, dissociated 7/4/8/4/9-atom or dissociated 7/4/8/4/8/4/9-atom core structures. This shows that Si-doping does not affect threading dislocation core structures in GaN. However, electron beam damage at $300 \mathrm{keV}$ produces 4-atom ring structures for $(a+c)$-type cores in Si-doped GaN.
\end{abstract}

Key words: Dislocation, Si doping, GaN, aberration-corrected STEM-HAADF, beam damage

\section{Introduction}

Group III nitride semiconductors are widely used in blue, green and ultraviolet light-emitting diodes (LEDs) and laser diodes (LDs) [1], and are of growing interest in high-electron mobility transistor applications. LEDs are based on a $p-n$ junction, where the majority of group III-nitride material in the device is doped either $n$-type (using $\mathrm{Si}$ ) or $p$-type (using $\mathrm{Mg}$ ). $\mathrm{GaN}$ films are usually grown on lattice-mismatched substrates (usually sapphire, $\mathrm{Si}$ or $\mathrm{SiC}$ ) 
[2], which produces a high density (as high as $5 \times 10^{9} \mathrm{~cm}^{-2}$ ) of threading dislocations (TDs) in these films. TDs which originate in the nucleation layer [2] near the GaN/sapphire substrate in our case, thread along [0001], through the Si-doped GaN junction, and into the active (InGaN) layer of the device. The efficiencies [1], lifetimes [3] and leakage currents [4] of GaN-based LED devices can be degraded severely by these one-dimensional crystal defects. Both microscopy [5] [6] [7] and theoretical predictions [8] have indicated that TDs in $n$-type doped GaN are negatively charged and can act as scattering centres in the material [8] [9] [10]. This is attributed to acceptor states that trap electrons and are introduced into the band gap by broken, under-coordinated or wrong bonds or by impurity segregation to the TD core [5] [7] [8] [10] [11].

It is therefore necessary to study both the core and near-core region of the dislocation to determine the core structure type and to investigate impurity segregation at TDs in the material, to better understand the electronic properties of dislocations in Si-doped GaN. High tensile stresses are also known to develop during the growth of Si-doped GaN layers, which can lead to cracking and difficulties in growth of device structures. The development of these stresses, although not fully understood, has been associated with the inhibition of dislocation climb [12], $\mathrm{SiN}_{\mathrm{x}}$ masking [13] [14], strain relaxation [15] [16], the interaction of bent dislocations with planar defects [17], surface roughness [18], electronic effects [19] [20] and surface-mediated climb [21]. Further study of dislocations is therefore needed to develop routes to overcome these technologically-important phenomena of carrier scattering and stress development in Si-doped GaN films.

The atomic arrangements at dislocation cores in GaN have been studied previously [22] [23] [24] [25] [26] [27]. Three types of TDs are found in GaN-based films and devices: those with $a$-type Burgers vectors (usually edge), $(a+c)$-type Burgers vectors (usually mixed) and $c$-type Burgers vectors (usually screw). For $a$-type dislocations in GaN, all recent theoretical and experimental work has revealed that the most stable core structure is one that includes adjacent 5- and 7-atom rings in the (0001) plane [23]. Previous experimental data from GaN films [23] also showed two $(a+c)$-type core structures, a double 5/6-atom ring structure and a dissociated core with two $(a+c) / 2$ partial dislocations joined by an extended planar stackingfault lying on the (11-20) plane with repeating 4/8 rings and a displacement vector $R=1 / 6$ [1ํ13] [27]. Furthermore, theoretical studies have predicted structures including 4- [28], 
5/7- [29] and 8- [30] atom rings as stable core structures for $a$-type dislocations in pure $\mathrm{InN}$ films.

As the majority of group-III nitride material in a device is doped $n$-type (using $\mathrm{Si}$ ) or $p$-type (using $\mathrm{Mg}$ ), it is important to study detailed core structures of TDs in doped GaN to understand the effect of dopant interaction with TDs. Although experimental studies [23] have been carried out to study dislocation core structures in Mg-doped GaN where all $a$-type TD cores formed 5/7-atom rings and all $(a+c)$-type TD cores observed were either double 5/6-atom or dissociated 7/4/8/4/9-atom rings, the study revealed that the dissociation of $(a+c)$-type threading dislocations was suppressed in $\mathrm{Mg}$-doped $\mathrm{GaN}$ due to the significant interaction of $\mathrm{Mg}$ with threading dislocations. On the other hand, no experimental studies on TD core structures in $n$-doped GaN films with doping levels similar to those used in devices are available in the literature.

Theoretical studies [31] [32] are available and predict that TD cores containing Ga vacancies (i.e. 8-atom ring structures with Ga atoms missing at the core) are the most stable for $a$-type dislocations in $n$-type $\mathrm{GaN}$ grown under nitrogen-rich conditions. Moreover, the only experimental study reported for $n$-type doped $\mathrm{GaN}$ was undertaken on a Si-doped sample with a medium doping level of $\sim 2 \times 10^{18}$ atoms $/ \mathrm{cm}^{3}$. The study reported a core structure with a single 8-atom ring in the (0001) plane for an a-type dislocation [33], although the authors later acknowledged that the dislocation was an $(a+c)$-type rather than an $a$-type dislocation [34]. The authors also correlated the resultant scanning transmission electron microscopy-high angle annular dark-field (STEM-HAADF) intensity at the dislocation core with the number of atoms at the column location. Their calculations predicted a Ga-vacancy concentration between 0 and $15 \%$ per $c$-axis repeat at the core. As devices contain Si-doping levels of $\sim 10^{19}$ atoms $/ \mathrm{cm}^{3}$ and medium doping levels of $2 \times 10^{18}$ atoms $/ \mathrm{cm}^{3}$ do not introduce as much tensile stress as observed in commercial devices, studies on medium-doped samples are not a true representation of the effect of Si-doping on TD core structures in actual GaN devices. Thus, we present a statistically-valid STEM-HAADF study comparing the core structures of 200 dislocations in undoped and Si-doped GaN (up to $1 \times 10^{19}$ atoms $/ \mathrm{cm}^{3}$ as used in devices) to determine the effect of Si-doping on dislocation core structures in $\mathrm{GaN}$.

All samples were grown in a Thomas Swan $6 \times 2$ " close-coupled showerhead metalorganic vapour phase epitaxy (MOVPE) reactor. Trimethylgallium (TMG), silane $\left(\mathrm{SiH}_{4}\right)$ and ammonia $\left(\mathrm{NH}_{3}\right)$ were used as precursors for $\mathrm{Ga}$, Si and $\mathrm{N}$, respectively. Four samples; high dislocation density (HDD) undoped, low dislocation density (LDD) undoped, LDD low Si- 
doped and HDD high Si-doped GaN samples were investigated in this study. Details of undoped and $\mathrm{Si}$-doped $\mathrm{GaN}$ epilayer growth on sapphire have been described in a previous publication [12]. Both Si-doped films included an initial $200 \mathrm{~nm}$ of undoped region, followed by an $800 \mathrm{~nm}$ region doped with $\mathrm{Si}$, to ensure that Si doping does not influence the nucleation layer or the island coalescence step during the early stages of film growth. The Si-dopant concentration was chosen to be as high as possible without causing cracking or surface roughening [35].

Electron-transparent TEM specimens were prepared by mechanical polishing, dimpling and back-thinning with $\mathrm{Ar}^{+}$ions at $5 \mathrm{keV}$, and final ion milling was performed at $2 \mathrm{keV}$ to minimize specimen damage. Plan-view STEM-HAADF imaging was then performed at 300 keV using a $\operatorname{Titan}^{3} 80-300$ aberration-corrected electron microscope, where at least fifty dislocations for each sample were observed for statistical reliability. This provided highquality images for TDs aligned parallel to the [0001] direction. However, some TDs were inclined away from the [0001] direction for the HDD high Si-doped sample (as in [27]) and as shown in the supplementary material [36], so that the cores could not be observed clearly in a single plan-view image. Therefore, for each of these TDs, a through-focal series technique was used to obtain a depth-dependent set of images in order to locate a region of the core without kinks or jogs, similar to the method followed by Lozano et al. [37] and Yang et al. [38]. This was done using an aberration-corrected Nion Ultrastem 100 TEM operated at $100 \mathrm{keV}$. All geometric aberrations in the electron beam probe were corrected up to the third order and fifth order for the $\operatorname{Titan}^{3}$ 80-300 and Nion UltrastemTM 100 TEM respectively. All experimental images were processed using an average background subtraction filter with a cycle of 20 and 5 steps. More details on the basic characterization of these films can be found in the supplementary materials [36].

Plan-view high-resolution STEM-HAADF images of TD cores acquired along the [0001] zone axis indicated that all $a$-type dislocations in all samples formed 5/7-atom ring cores, independent of growth conditions or Si-doping (as shown in Figure 1). The $a$-type dislocations could be distinguished clearly from $(a+c)$-type dislocations, as the latter show surface relaxation due to the Eshelby Twist of the lattice [37].

In addition, in all samples, $50 \%$ of $(a+c)$-type dislocations dissociated into extended core structures (e.g. $7 / 4 / 8 / 4 / 8 / 4 / 9$ - or 7/4/8/4/9-atom rings), whereas the rest retained the 
undissociated double 5/6-atom ring core structure (as shown in Figure 1), consistent with previous theoretical predictions and corresponding STEM-HAADF images acquired for undoped GaN [23] (full details in Table 1). Si-doping produced no detectable differences in dislocation core structures for either $a$-type or $(a+c)$-type dislocations in all undoped and $\mathrm{Si}$ doped $\mathrm{GaN}$ samples.

In addition to the statistically-valid HRSTEM-HAADF study, molecular dynamics (MD) simulations were also performed on the HDD high Si-doped GaN film to investigate the effect of the tensile stress introduced by Si-doping on TD core structures in GaN. The application of an in-plane tensile stress (of $1.18 \mathrm{GPa}$ calculated using the modified Stoney's formula [39] on in-situ wafer curvature measurements reported previously by Moram et al. [12]) to large simulation cells was not found to alter the core morphology and resulting core structures. A detailed explanation of the technique is given in the supplementary material [36]. Thus, both MD simulations and the statistically-valid HRSTEM-HAADF study show that even high Si-doping levels of $\sim 1 \times 10^{19}$ atoms $/ \mathrm{cm}^{3}$ (as used in devices) do not affect TD core structures in GaN.

Although $a$-type TDs have previously been observed to have the 5/7-atom ring structure for GaN-based films, independent of threading dislocation density [23], Mg-doping level [23] and In-alloying [40], previous studies have shown that different Mg-doping and In-alloying levels affect the stability of $(a+c)$-type cores in $\mathrm{GaN}$ [23] [40]. This is because the solutedislocation interaction is also an important factor that affects TD core structures in doped and alloyed GaN. Therefore, initial impurity-dislocation binding energy calculations according to the model of Fiore and Bauer [41] were also performed to predict whether silicon interacts with TD cores in Si-doped GaN. The relative degree of Si segregation to the strain fields around dislocations in GaN with a Burgers vector containing an $a$-component was estimated (as shown in Figure 2), assuming that the relative difference between the radii of $\mathrm{Si}_{\mathrm{Ga}}{ }^{+}$compared to that of $\mathrm{Ga}$ is the main driver of $\mathrm{Si}$ segregation. Figure 2 shows an approximate two-fold increase in the $\mathrm{Si}$ concentration at a distance of $0.3 \mathrm{~nm}\left(\mathbf{b}_{\mathrm{e}}\right)$, and perpendicular to the Burgers vector, assuming a background Si concentration of $1 \times 10^{19}$ atoms $/ \mathrm{cm}^{3}$ occurring at a film growth temperature of $1050{ }^{\circ} \mathrm{C}$. Since these binding energies are predicted to be sufficiently larger than the thermal energy available during growth, this result strongly suggests that there is a high probability of Si substituting for Ga near the compressive region of the TD core with an $a$-type component consisting of both tensile and 
compressive lobe regions, thereby validating the work of Hull and Bacon [42] which states that impurities/solute atoms with size lower than that of solvent atoms segregate to the compressive lobe of an edge dislocation.

Although our calculations predict a high probability of solute atom-dislocation interaction in the high Si-doped GaN sample, our STEM-HAADF results clearly show that both $a$ - and $(a+c)$-type TD core structures are unaffected by high Si-doping. This means that although segregation of $\mathrm{Si}$ or $\mathrm{Si}$-atmosphere formation at $(a+c)$-type TD cores is highly probable, any change in the core structures in $\mathrm{GaN}$ is inhibited. This could be attributed to the inhibition of climb caused by Si-atmosphere formation at TDs with $a$-components, as previously predicted by Moram et al. [12]. Si-atmosphere formation could inhibit TD climb, prevent strain relief, and thus result in high tensile stresses in Si-doped $\mathrm{GaN}$ [12]. Although it can be argued that the calculated additional tensile stress of $1.18 \mathrm{GPa}$ introduced by Si-doping should drive $(a+c)$-type TD core dissociation in Si-doped $\mathrm{GaN}$, the dissociation of $(a+c)$-type TD cores into $1 / 2(a+c)$-partials is climb-driven. Therefore $\mathrm{Si}$-atmosphere formation at TD cores inhibits the climb-driven dissociation, resulting in all $(a+c)$-type core structures in the top $800 \mathrm{~nm}$ of the high Si-doped GaN film exhibiting the same core structures as in the $200 \mathrm{~nm}$ of the undoped GaN film on which it was grown. This explains why Si-doping does not affect the TD core structures in GaN.

Furthermore, evidence of beam damage affecting $(a+c)$-type TD core structures at $300 \mathrm{keV}$ was also observed by our study (as shown in Figure 3), where an $(a+c)$-type core in the HDD high Si-doped GaN sample changed from a double 5/6-atom to a 4-atom ring after $25 \mathrm{~s}$ of beam exposure. Our observations are in line with a previous study where an $a$-type $(\mathbf{b}=1 / 3<$ $11 \overline{2} 0>$ ) core exhibited glide motion (on the $(0001)$ basal- and the $<1 \overline{1} 0 n>$ pyramidal-plane) at room temperature under the influence of the electron beam used for TEM observations [11]. As 4-atom cores form by the glide motion of an 8-atom (or double 5/6-atom) core in $\mathrm{GaN}$ [43], glide introduced by the $300 \mathrm{keV}$ electron beam could easily change the core structure of $(a+c)$-type TD cores from 8- (double 5/6-atom rings) to 4-atom rings as shown in Figure 3 (c), (g) and (h) which were acquired after 35, 45 and $155 \mathrm{~s}$ of $300 \mathrm{keV}$ beam exposure. Moreover, beam damage could also result in $\mathrm{N}$-deficient or non-stoichiometric cores, similar to the structure of 4-atom rings. Further investigation is required to understand the core structure type obtained after prolonged beam damage at $300 \mathrm{keV}$. As TDs in the HDD high Si-doped GaN sample were tilted, it was necessary to study these structures by the optical 
sectioning technique [37] [38] which resulted in cumulative beam exposures of up to $150 \mathrm{~s}$ to the TD core. This is in contrast with small beam exposures of up to $10 \mathrm{~s}$ for each TD core imaged by HRSTEM-HAADF in the statistically-valid study undertaken on undoped GaN [23], as only one or two images of each TD core were acquired to minimise electron dosage time to below $10 \mathrm{~s}$ to limit any beam damage to the core.

Our results show that even high Si-doping levels do not affect TD core structures in GaN even though our binding energy calculations clearly suggest that $\mathrm{Si}$ segregates to the $a$ components of TDs in GaN. This is attributed to TD climb inhibition caused by Siatmosphere formation at TD cores. High Si-doping not affecting TD core structures in GaN is further evidence that Si segregates to TD cores, forms atmospheres which inhibit climb and prevent strain relief, which ultimately results in high tensile stresses and cracking observed in thick highly Si-doped GaN films. Moreover, prolonged $300 \mathrm{keV}$ electron beam exposure of $(a+c)$-type cores results either in non-stoichiometric cores (resembling 4-atom rings) or electron beam induced TD glide forming 4-atom $(a+c)$-type cores. Thus, prolonged beam damage is an important parameter to be considered while conducting statistically-valid studies on TD core structures in GaN.

\section{Acknowledgements}

This work was funded in part by the Cambridge Commonwealth trust, St. John's College, British Federation of Women Graduates and the EPSRC. MAM acknowledges support from the Royal Society through a University Research Fellowship. Additional support was provided by the EPSRC through the UK National Facility for Aberration-Corrected STEM (SuperSTEM).

\section{References}

[1] S. Nakamura, Jpn. J. Appl. Phys. 30, L1705 (1991).

[2] M. A. Moram, C. S. Ghedia, D. V. S. Rao, J. S. Barnard, Y. Zhang, M. J. Kappers, C. J. Humphreys, J. Appl. Phys., 106, 073513, 2009.

[3] M. F. Schubert, J. Xu, J. K. Kim, E. F. Schubert, M. H. Kim, S. Yoon, S. M. Lee, C. Sone, T. Sakong, and Y. Park, Appl. Phys. Lett. 93, 041102 (2008).

[4] J. W. P. Hsu, M. J. Manfra, R. J. Molnar, B. Heying, and J. S. Speck, Appl. Phys. Lett. 81, 79 (2002).

[5] D. Cherns and C. G. Jiao, Phys. Rev. Lett., 87, 205504, 2001. 
[6] G. Koley and M. G. Spencer, Appl. Phys. Lett., 78, 2873, 2001.

[7] J. Cai and F. A. Ponce, Phys. Stat. Sol. (a), 192, 407, 2002.

[8] D. C. Look and J. R. Sizelove, Phys. Rev. Lett., 82, 1237, 1999.

[9] N. G. Weinmann, L. F. Eastman, D. Doppalapudi, H. M. Ng, T. D. Moustakas, J. Appl. Phys., 83, 3656, 1998.

[10] H. M. Ng, D. Doppalapudi, T. D. Moustakas, N. G. Weinmann, L. F. Eastman, Appl. Phys. Lett., 73, 821, 1998.

[11] K. Maeda, K. Suzuki, M. Ichihara, S. Nishiguchi, K. Ono, Y. Mera, S. Takeuchi, Physica B: Condensed Matter, 273, 13415, 1999.

[12] M. A. Moram, M. J. Kappers, F. Massabuau, R. A. Oliver, C. J. Humphreys, J. Appl. Phys., 109, 073509, 2011.

[13] A. Dadgar, P. Veit, F. Schulze, J. Bläsing, A. Krtschil, H. Witte, A. Diez, T. Hempel, J. Christen, R. Clos, A. Krost, Thin Solid Films, 515, 4356, 2007.

[14] A. Dadgar, J. Bläsing, A. Diez, A. Krost, Appl. Phys. Express, 4, 011001, 2011.

[15] P. Cantu, F. Wu, P. Waltereit, S. Keller, A. E. Romanov, U. K. Mishra, S. P. DenBaars, J. S. Speck, J. Appl. Phys., 97, 103534, 2005.

[16] A. E. Romanov, G. E. Beltz, P. Cantu, F. Wu, S. Keller, S. P. DenBaars, J. S. Speck, Appl. Phys. Lett., 89, 161922, 2006.

[17] J. S Sánchez, F. J. Pacheco, J. M. Calleja, M. A. Sánchez-Garcia, E. Calleja, Appl. Phys. Lett., 78, 4124, 2001.

[18] D. M. Follstaedt, S. R. Lee, A. A. Allermn, J. A. Floro, J. Appl. Phys., 105, 083507, 2009.

[19] J. Xie, S. Mita, L. Hussey, A. Rice, J. Tweedie, J. LeBeau, R. Colllazo, Z. Sitar, Appl. Phys. Lett., 98, 202101, 2011.

[20] J. Xie, S. Mita, L. Hussey, A. Rice, J. Tweedie, J. LeBeau, R. Colllazo, Z. Sitar, Appl. Phys. Lett., 99, 141916, 2011.

[21] K. Forghani, L. Schade, U. T. Schwarz, F. Lipski, O. Klein, U. Kaiser, F. Scholz, J. Appl. Phys., 112, 093102, 2012.

[22] L. Lymperakis, J. Neugebauer, M. Albrecht, T. Remmele, and H. P. Strunk, Phys. Rev. Lett., 93, 196401, 2004.

[23] S. K. Rhode, M. K. Horton, M. J. Kappers, S. Zhang, C. J. Humphreys, R. O. Dusane, S.-L. Sahonta, M. A. Moram, Phys. Rev. Lett., 111, 025502, 2013.

[24] P. Ruterana, V. Potin, D. Cherns, W. T. Young, and J. W. Steeds, Mater. Res. Soc. Symp. Proc. 482, 459 (1998). 
[25] Y. Xin, S. J. Pennycook, N. D. Browning, P. D. Nellist, S. Sivananthan, F. Omnès, B. Beaumont, J. P. Faurie, and P. Gibart, Appl. Phys. Lett. 72, 2680 (1998).

[26] I. Arslan, A. Bleloch, E. A. Stach, and N. D. Browning, Phys. Rev. Lett. 94, 025504 (2005).

[27] P. B. Hirsh, J. G. Lozano, S. Rhode, M. K. Horton, M. A. Moram, S. Zhang, M. J. Kappers, C. J. Humphreys, A. Yasuhara, E. Okunishi, P. D. Nellist, Phil. Mag., 93, $3925,2013$.

[28] H. P. Lei, R. Ruterana, G. Nouet, X. Y. Jiang, J. Chen, Appl. Phys. Lett., 90, 111901, 2007.

[29] J. Kioseoglou, Ph. Komninou, Th. Karakostas, Phys. Stat. Sol. (a), 206, 1931, 2009.

[30] J. Kioseoglou, Phys. Stat. Sol. (a), 210, 204, 2013.

[31] A. F. Wright and U. Grossner, Appl. Phys. Lett., 73, 2751, 1998.

[32] K. Leung, A. F. Wright, E. B. Stechel, Appl. Phys. Lett., 74, 2495, 1999.

[33] Y. Xin, E. M. James, I. Arslan, S. Sivananthan, N. D. Browning, S. J. Pennycook, F. Omnès, B. Beaumont, J.-P. Faurie, P. Gibart, Appl. Phys. Lett., 76, 466, 2000.

[34] I. Arslan, A. Bleloch, E. A. Stach, S. Ogut, N. D. Browning, Phil. Mag., 86, 4727, 2011.

[35] P. Vennéguès, M. Leroux, S. Dalmasso, M. Benaissa, P. De Mierry, P. Lorenzini, B. Damilano, B. Beaumont, J. Massies, P. Gibart, Phys. Rev. B, 68, 235214, 2003.

[36] See supplemental material at [URL will be inserted by AIP] for basic characterization of Si-doped films.

[37] J. G. Lozano, H. Yang, M. P. Guerrero-Lebrero, A. J. D’Alfonso, A. Yasuhara, E. Okunishi, S. Zhang, C. J. Humphreys, L. J. Allen, P. L. Galindo, P. B. Hirsch, P. D. Nellist, Phys. Rev. Lett., 113, 135503, 2014.

[38] H. Yang, J. G. Lozano, T. J. Pennycook, L. Jones, P.B. Hirsch, P. D. Nellist, Nature Commun., 6, 7266, 2015.

[39] C. A. Klein, Comment, J. Appl. Phys., 88, 5499, 2000.

[40] Sneha K. Rhode*, M. K. Horton", S. -L. Sahonta, M. J. Kappers, S. J. Haigh, T. J. Pennycook, C. McAleese, C. J. Humphreys, R. O. Dusane, M. A. Moram, J. Appl. Phys., submitted, 2015.

[41] N. F. Fiore and C.L. Bauer, Prog. Mater. Sci., 13, 85, 1968.

[42] D. Hull and D. J. Bacon, Introduction to dislocations, 2001.

[43] A. Béré, A. Serra, Phil. Mag., 86, 2159, 2006.

[44] R. A. Oliver, M. J. Kappers, J. Sumner, R. Datta, C. J. Humphreys, J. Cryst. Growth, 289, 506, 2006. 
[45] M. A. Moram, T. C. Sadler, M. Häberlen, M. J. Kappers, C. J. Humphreys, Appl. Phys. Lett., 97, 261907, 2010.

[46] J. D. Accord, I. C. Manning, X. Weng, D. W. Snyder, J. M. Redwing, Appl. Phys. Lett., 93, 111910, 2008.

[47] M. K. Horton, S. K. Rhode, M. A. Moram, J. Appl. Phys., 116, 063710, 2014.

[48] A. Stukowski, Simul. Mater. Sci. Eng., 18, 015012, 2010.

\begin{tabular}{|c|c|c|c|c|}
\hline & $\begin{array}{c}5 / 7 \text {-atom ring } \\
a \text {-cores } \%\end{array}$ & $\begin{array}{c}\text { Dissociated } \\
(a+c) \text {-cores \% }\end{array}$ & $\begin{array}{c}\text { Double 5/6- } \\
(a+c) \text {-cores \% }\end{array}$ & $\begin{array}{c}\text { Dislocation } \\
\text { lengths (nm) }\end{array}$ \\
\hline LDD undoped & 100 & $50 \pm 5$ & $50 \pm 5$ & $1.85 \pm 0.06$ \\
\hline LDD Si-doped & 100 & $54 \pm 9$ & $48 \pm 9$ & $1.76 \pm 0.05$ \\
\hline HDD undoped & 100 & $50 \pm 5$ & $50 \pm 5$ & $1.62 \pm 0.05$ \\
\hline HDD Si-doped & 100 & $43 \pm 3$ & $57 \pm 3$ & $1.59 \pm 0.03$ \\
\hline
\end{tabular}

Table 1: The total percentage of dissociated and undissociated $(a+c)$-type dislocations, and the dissociation lengths of the dissociated $(a+c)$-type dislocations in both HDD and LDD undoped and Si-doped GaN samples. 


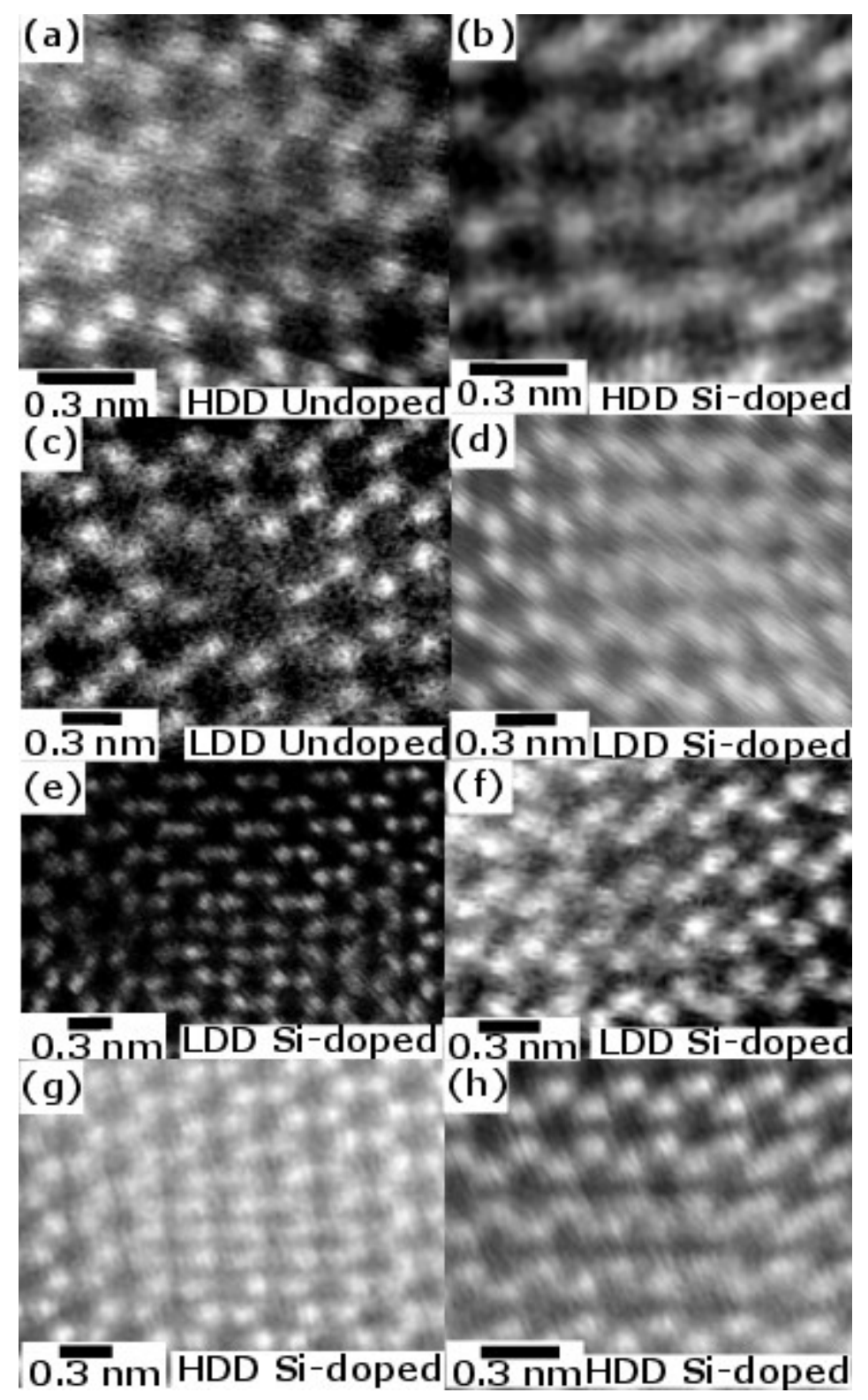

Figure 1: Typical plan-view STEM-HAADF images for (a) 5/7-atom ring $a$-type TD cores acquired along the [0001] zone axis of HDD undoped GaN and (b) high Si-doped GaN, and LDD (c) undoped GaN and (d) low Si-doped GaN, (e) $(a+c)$-type 7/4/8/4/8/4/9-atom dissociated core and (f) double 5/6-atom undissociated core in LDD low Si-doped GaN. (g) Typical plan-view STEM-HAADF images from a through-focal series acquired for an $(a+c)$ type 7/4/8/4/9-atom dissociated and (h) double 5/6-atom undissociated $(a+c)$-type core in HDD high Si-doped GaN. The bright dots in all figures correspond to atomic columns containing both $\mathrm{Ga}$ and $\mathrm{N}$ atoms. 


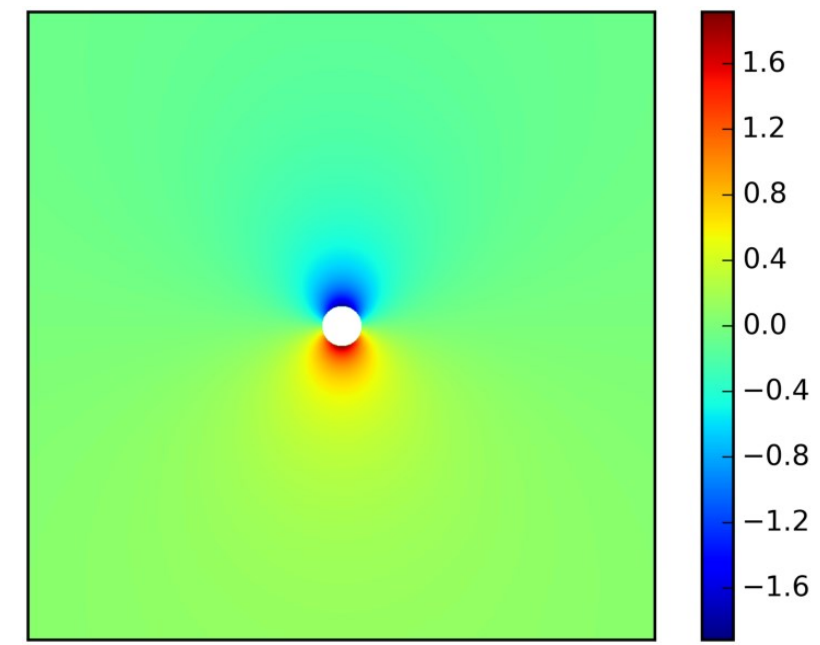

Figure 2: $10 \mathrm{~nm} \times 10 \mathrm{~nm}$ section in the (0001) plane around a dislocation with an $a$ component showing the relative Si concentration profile using initial impurity-dislocation binding energy calculations, where 0 denotes the bulk Si concentration of $1 \times 10^{19}$ atoms $/ \mathrm{cm}^{3}$. The white circle in the centre has a radius of $1 \mathbf{b}_{\mathrm{e}}$ and indicates the region where the analytical expressions used in the model no longer apply. The colour scale shows the relative Si concentration. 

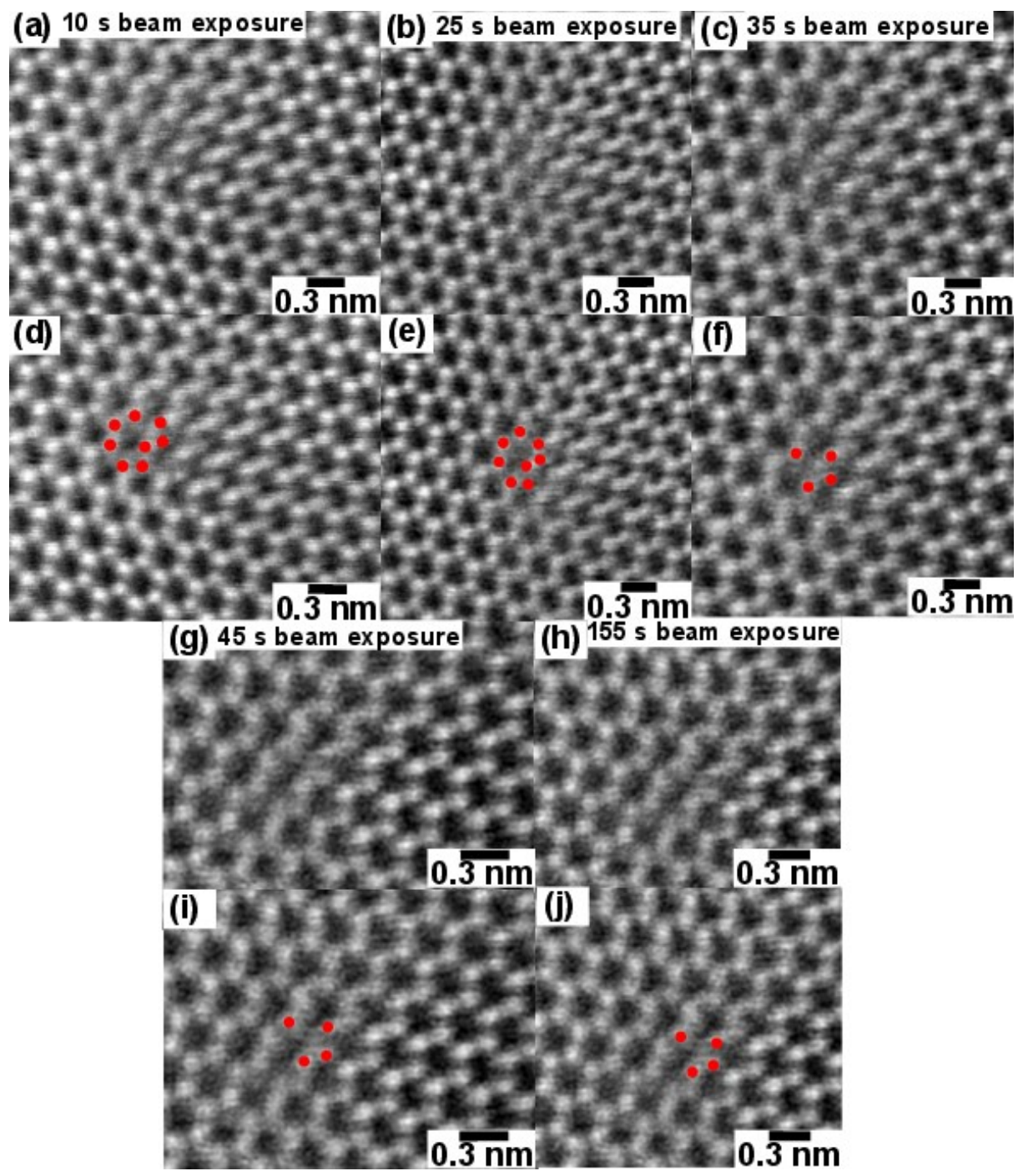

Figure 3: Typical plan-view STEM-HAADF images acquired for an $(a+c)$-type dislocation core from a single focal-series stack acquired at $300 \mathrm{keV}$ for an HDD high Si-doped GaN sample showing a (a) double 5/6-atom undissociated core after $10 \mathrm{~s}$ beam exposure, (b) a damaged double 5/6-atom core after $25 \mathrm{~s}$ electron beam exposure, (c) 4-atom ring after $35 \mathrm{~s}$ electron beam exposure, (g) 4-atom ring after $45 \mathrm{~s}$ of beam exposure and (h) 4-atom ring after 155 s of beam exposure. (d), (e), (f), (i) and (j) show the corresponding images shown in (a), (b), (c), (g) and (h) with the dislocation core highlighted. 
